\title{
Post-Translational 0xidative Modification and Inactivation of Mitochondrial Complex I in Epileptogenesis
}

\author{
Kristen Ryan, Donald S. Backos, Philip Reigan, and Manisha Patel \\ Department of Pharmaceutical Sciences, University of Colorado Anschutz Medical Campus, Aurora, Colorado 80045
}

Mitochondrial oxidative stress and damage have been implicated in the etiology of temporal lobe epilepsy, but whether or not they have a functional impact on mitochondrial processes during epilepsy development (epileptogenesis) is unknown. One consequence of increased steady-state mitochondrial reactive oxygen species levels is protein post-translational modification (PTM). We hypothesize that complex I (CI), a protein complex of the mitochondrial electron transport chain, is a target for oxidant-induced PTMs, such as carbonylation, leading to impaired function during epileptogenesis. The goal of this study was to determine whether oxidative modifications occur and what impact they have on CI enzymatic activity in the rat hippocampus in response to kainate (KA)-induced epileptogenesis. Rats were injected with a single high dose of KA or vehicle and evidence for CI modifications was measured during the acute, latent, and chronic stages of epilepsy. Mitochondrial-specific carbonylation was increased acutely ( $48 \mathrm{~h})$ and chronically $(6$ week), coincident with decreased CI activity. Mass spectrometry analysis of immunocaptured CI identified specific metal catalyzed carbonylation to Arg76 within the $75 \mathrm{kDa}$ subunit concomitant with inhibition of CI activity during epileptogenesis. Computational-based molecular modeling studies revealed that Arg76 is in close proximity to the active site of CI and carbonylation of the residue is predicted to induce substantial structural alterations to the protein complex. These data provide evidence for the occurrence of a specific and irreversible oxidative modification of an important mitochondrial enzyme complex critical for cellular bioenergetics during the process of epileptogenesis.

\section{Introduction}

Mitochondrial oxidative stress and resultant dysfunction has been implicated as a contributing factor in diverse acute and age-related chronic neurodegenerative disorders, including amyotrophic lateral sclerosis, Parkinson's disease (PD), Huntington's disease, and Alzheimer's disease (Schapira, 1999; Beal, 2005). However, the role of mitochondrial oxidative stress in the etiology of epilepsies is unknown. Mitochondria execute crucial functions that can impact neuronal excitability. While a small percentage of inherited epilepsies are associated with mitochondrial dysfunction, less is known about its role in acquired epilepsies such as temporal lobe epilepsy (TLE), which is characterized by initial trauma triggering complex molecular, biochemical, physiological, and structural changes in the brain culminating in spontaneous and recurrent seizures via a process termed epileptogenesis. Several key features of TLE, such as its progressive nature and metabolic demands, suggest mitochondrial decline in its eti-

Received Feb. 22, 2012; revised June 22, 2012; accepted July 1, 2012.

Author contributions: K.R. and M.P. designed research; K.R. and D.S.B. performed research; D.S.B. and P.R. contributed unpublished reagents/analytic tools; K.R. and D.S.B. analyzed data; K.R. and M.P. wrote the paper.

This work is supported by National Institutes of Health Grant R01NS039587. We thank Dr. Yogendra Raol and the University of Colorado Anschutz Medical Campus (UCAMC) In Vivo Neurophysiology Core facility, Mr. Joe Gomez and the UCAMC Mass Spectrometry Core facility, and the Computational Chemistry and Biology Core facility at the UCAMC School of Pharmacy, as well as the Proteomics Core at National Jewish Health in Denver, Colorado, for their technical assistance.

Correspondence should be addressed to Dr. Manisha Patel, Skaggs School of Pharmacy and Pharmaceutical Sciences, University of Colorado Anschutz Medical Campus, 12850 East Montview Boulevard, Aurora, CO 80045. E-mail: manisha.patel@ucdenver.edu.

DOI:10.1523/JNEUROSCI.0907-12.2012

Copyright $\odot 2012$ the authors $\quad 0270-6474 / 12 / 3211250-09 \$ 15.00 / 0$ ology. Recent work from our laboratory has demonstrated increased production of mitochondrial reactive oxygen species (ROS) during epileptogenesis in two distinct animal models (Jarrett et al., 2008; Waldbaum et al., 2010).

An important mechanism by which ROS can alter macromolecule function is via reversible or irreversible posttranslational modification (PTM) of critical proteins that can result in loss of function (Andersen, 2004; Dalle-Donne et al., 2006; Danielson and Andersen, 2008). The goal of the current study was to (1) determine whether increased ROS production observed during epileptogenesis results in PTMs of critical mitochondrial proteins and (2) if so, identify the functional consequence of the modification(s) using an in vivo rat model of TLE. Explicitly, we determined whether mitochondrialspecific oxidant-induced PTMs could be observed and if specific protein targets could be identified during the process of epileptogenesis.

Our results indicate that the $75 \mathrm{kDa}$ subunit of the mitochondrial electron transport chain (ETC) complex I (CI) is a target for oxidant-induced carbonylation during epileptogenesis. Arg76 of the $75 \mathrm{kDa}$ subunit was converted to a reactive glutamic semialdehyde (GSA) product through metal catalyzed oxidation (MCO), which coincided with decreased CI activity at acute and chronic stages of epileptogenesis. Computational-based molecular modeling simulations revealed that Arg76 is located proximal to the active site of CI and its carbonylation leads to conformational changes in both the adjacent subunits compromising the active site and iron-sulfur (Fe-S) centers involved in electron transport, which have the potential to negatively impact protein function. 


\section{Materials and Methods}

All materials were obtained from Sigma Aldrich or Fisher Scientific unless otherwise noted.

Induction of epileptogenesis. Animal housing was conducted in compliance with the University of Colorado Anschutz Medical Campus. Male Sprague Dawley rats $(\sim 300-350 \mathrm{~g})$ were subcutaneously administered saline (vehicle) or $11 \mathrm{mg} / \mathrm{kg}$ kainate (KA) (Nanocs KA-0002) dissolved in sterile buffered saline, $\mathrm{pH}$ 7.4. Animals were observed for status epilepticus (SE) and given moistened chow with subcutaneous saline injections $24 \mathrm{~h}$ later to decrease mortality, which was $10-15 \%$. Rats were killed by $\mathrm{CO}_{2}$ inhalation followed by a secondary method, i.e., immediate decapitation with a guillotine suitable for adult rats at $48 \mathrm{~h}, 1$ week, and 6 weeks after injection to encompass the acute, latent, and chronic phases of epileptogenesis. Hippocampi, cortex, and cerebellum were recovered from these animals, immediately frozen, and stored at $-80^{\circ} \mathrm{C}$ or crude mitochondria were isolated and immediately prepared for biochemical and mass spectrometry (MS) analysis.

Monitoring of convulsive seizures. Behavioral seizures during SE were evaluated by direct observation for $6 \mathrm{~h}$ after KA injection and scored based on a modified Racine scale (Racine, 1972) with only motor seizures being considered (Class I and II seizures were not scored). Briefly, motor seizure severity was characterized as follows: Class III animals displayed forelimb clonus with a lordotic posture, Class IV animals reared with concomitant forelimb clonus, and Class V animals had a Class IV seizure and loss of balance. All animals having at least three Class III convulsive seizures each hour for at least $3 \mathrm{~h}$ are included in the study. If KA-injected animals did not meet the criteria for SE (i.e., <Class III seizures), they were labeled as nonresponders. Direct observation confirmed that animals were not having convulsive seizure activity $48 \mathrm{~h}$ and 1 week post-KA treatment (data not shown). Rats were monitored by $24 / 7$ video in the University of Colorado In Vivo Neurophysiology Core for seizure number, duration, and severity during the 6 week time point to confirm epilepsy. Rats observed to have $\geq 2$ spontaneous and convulsive seizures during the 6 week time point were considered epileptic.

Isolation of brain mitochondria and synaptosomes. Crude hippocampal, cortical, and cerebellar mitochondria were isolated from $50 \mathrm{mg}$ tissue according to our previous methods (Liang and Patel, 2006). The remaining pellet (crude mitochondria) was collected and used immediately for $\mathrm{CI}$ immunoprecipitation. Also, crude mitochondria were frozen at $-80^{\circ} \mathrm{C}$ for CI activity measurements or Western blot analysis. For all studies, one hippocampus was used for each experiment with the exception of immunoprecipitation of CI subunits, in which both hippocampi from each rat were used. Synaptosomes were isolated according to our previous method (Liang et al., 2012). Synaptosomes were resuspended in $25 \mathrm{~mm}$ potassium phosphate buffer $+5 \mathrm{~mm} \mathrm{MgCl}_{2}, \mathrm{pH} 7.0$, for measurement of CI activity.

Biotin-hydrazide detection of carbonylated proteins. Isolated crude mitochondria or immunoprecipitated CI proteins from saline and KAtreated rats were cleared for $1 \mathrm{~h}$ with NeutrAvidin beads (Pierce) before treatment with $5 \mathrm{~mm}$ biotin-hydrazide (Pierce) for $2 \mathrm{~h}$ rotating in the dark (Codreanu et al., 2009; Galligan et al., 2012). Samples were then reduced with $10 \mathrm{~mm} \mathrm{NaBH}_{4}$ for $1 \mathrm{~h}$ in the dark before separation by SDS-PAGE. For crude mitochondria, $30 \mu \mathrm{g}$ protein (Bradford assay) from three separate animals per time point was loaded onto a $10 \%$ Tris$\mathrm{HCl}$ Ready gel (Bio-Rad). Samples were separated and transferred to a polyvinylidene difluoride membrane with a $0.45 \mu \mathrm{m}$ pore size (Millipore). After blocking the membrane in 5\% nonfat dry milk (Bio-Rad), carbonylation was detected by membrane incubation with a biotinlinked horseradish peroxidase primary antibody for $1 \mathrm{~h}$ (GeneTex) at 1:7500 and developed with Enhanced Chemiluminesence Plus reagents (GE Healthcare Biosciences). Bands were visualized with a Storm Optical Scanner (Molecular Dynamics) and band densitometry was quantified with National Institutes of Health (NIH) ImageJ 1.44 software and values were calculated as percentage to control (\% Control). For samples of immunoprecipitated CI subunits (see below, CI immunocapture method), $10-20 \mu \mathrm{g}$ of protein was derivitized with $5 \mathrm{~mm}$ biotin-hydrazide for $2 \mathrm{~h}$ rotating in the dark and then reduced with $10 \mathrm{mM} \mathrm{NaBH}_{4}$ for $1 \mathrm{~h}$ in the dark before separation by SDS-PAGE. Rats that did not meet criteria for
KA-induced SE (see criteria above for nonresponders) were also prepared for carbonylation assessment in an identical manner.

$C I$ and CIV activity. NADH dehydrogenase (CI) and cytochrome $c$ oxidase (CIV) activity was measured as described previously (BirchMachin and Turnbull, 2001; Tieu et al., 2003). CI activity was measured in $1 \mathrm{ml}$ reactions with $100 \mu \mathrm{g}$ of crude hippocampal mitochondria by the oxidation of $\mathrm{NADH}$ at $340 \mathrm{~nm}$ for $2 \mathrm{~min}$ at $30^{\circ} \mathrm{C}$ with a Shimatzu UV2401PC spectrophotometer. The average CI activity for Ctl samples was calculated to be $148.1 \pm 4.421 \mathrm{U} / \mathrm{min} / \mathrm{mg}$ protein. Ctl values were normalized to 100 and data are represented as percentage control (\% Control). CIV activity was measured in $1 \mathrm{ml}$ reactions with $25 \mu \mathrm{g}$ crude hippocampal mitochondria by the oxidation of cytochrome $c$ (reduced with ascorbic acid) at $550 \mathrm{~nm}$ for $45 \mathrm{~s}$ at $30^{\circ} \mathrm{C}$.

CI immunocapture. CI was isolated according to Schilling et al. (2005) to assess PTMs after KA treatment. Briefly, $300 \mu \mathrm{g}$ of crude hippocampal mitochondria from saline or KA-treated rats was solubilized in cold PBS containing $1 \% N$-dodecyl- $\beta$-D-maltoside (DDM) for $30 \mathrm{~min}$ on ice for isolation of membrane proteins. Samples were then centrifuged at 20,000 $g$ for $30 \mathrm{~min}, 4^{\circ} \mathrm{C}$ and supernatants were incubated overnight with agarose beads irreversibly cross-linked to specific monoclonal antibodies for CI proteins (Mitosciences). CI proteins were eluted from the beads with $30 \mu \mathrm{l} 0.1 \mathrm{M}$ glycine, $\mathrm{pH} 2.5$, supplemented with $0.05 \%$ DDM. For biotinhydrazide derivatization experiments, proteins were eluted with $30 \mu \mathrm{l}$ $1 \%$ SDS. Protein concentration was determined on a NanoDrop 2000 before the addition of $30 \mu \mathrm{l} 2 \times$ SDS-PAGE loading buffer (Bio-Rad) and $5 \mu \mathrm{l} 1 \mathrm{M}$ Tris, $\mathrm{pH} 8.0$, neutralization buffer. CI proteins were resolved by SDS-PAGE on a $4-20 \%$ Tris-HCl Ready gel. Gels were then stained for band excision (described below) and trypsin digest.

Protein band excision and protein identification peptide mass fingerprinting. Following SDS-PAGE, CI protein bands were identified with Imperial Protein Stain (Pierce) according to manufacturers' instructions and were excised with sterile razor blades. Gel bands were transferred to a $0.5 \mathrm{ml}$ tube and were digested by "in-gel" cleavage with a modified protocol (Shevchenko et al., 2006). Briefly, gel slices were reduced with $10 \mathrm{~mm}$ dithiothreitol, alkylated with $25 \mathrm{~mm}$ iodoacetamide, and finally digested overnight in $0.3 \mu \mathrm{g}$ trypsin (Promega V5111) at $37^{\circ} \mathrm{C}$. Peptides were extracted from gel pieces in a sonicating waterbath first with 1:1 acetonitrile: $50 \mathrm{~mm} \mathrm{NH}_{4} \mathrm{HCO}_{3}$ then $0.1 \%$ formic acid before drying completely in a SpeedVac (Savant AES1000) at $37^{\circ} \mathrm{C}$ for $40 \mathrm{~min}$. Peptides were then resolubilized in $100 \mathrm{~mm}$ acetic acid. For protein identification via peptide mass fingerprinting (PMF) the peptides were extracted, desalted using C18 Ziptips (Millipore), and mixed with matrix $(10 \mathrm{mg} / \mathrm{ml}$ $\alpha$-cyano-4-hydroxycinnamic acid in $60 \%$ acetonitrile/ $0.1 \%$ trifluoroacetic acid) at a ratio of $1: 1$ and then spotted in $1 \mu \mathrm{l}$ aliquots onto a target plate and analyzed by MALDI-TOF MS on a Bruker Omniflex with Flex Control Software 2.0. Obtained mass spectra were externally calibrated with an equimolar mixture of bradykinin fragment, angiotensin II, $\mathrm{P}_{14} \mathrm{R}$, ACTH 18-39, and insulin oxidized B chain (ProteoMass Kit; MSCAL1Sigma). Spectra were analyzed with Flex Analysis Software 2.0 and database searches were conducted with BioTools 2.2 using Mascot 2.2 (Matrix Science; http://www.matrixscience.com). Protein identification (ID) parameters included optional modifications to methionine (oxidized) and cysteine (carbamidomethyl), SwissProt database, and Rattus taxonomy. Mascot scores were used to determine significant protein identification. Five or more peptides identified per protein were necessary for significant IDs. Samples were run and data analysis performed at the Skaggs Colorado School of Pharmacy and Pharmaceutical Sciences Mass Spectrometry Core at the University of Colorado Anschutz Medical Campus.

Detection of protein carbonylation by quantitative time-of-flight MS. Once the $75 \mathrm{kDa}$ subunit of CI was accurately identified by PMF, we assessed samples for protein carbonylation modifications. Peptides were resolubilized in 3\% acetonitrile $/ 0.1 \%$ formic acid and directly analyzed on an Agilent 6340 Ion Trap with LC chip cube. MS/MS data were analyzed in SpectraMill (Rev A.03.03.084 SR4). Protein identification parameters included optional modifications to methionine (oxidized; +16 Da mass shift), cysteine (carbamidomethyl; +57 Da mass shift), and arginine (GSA; -43 Da mass shift). The SwissProt database was searched with Rattus taxonomy and allowance of two missed cleavages. MS/MS 
spectral features were set at $\mathrm{MH}+=200-4000 \mathrm{Da}$, the minimum M/S signal-to-noise was set to 10 and the maximum at $z=4$, and the precursor mass tolerance for the quantitative time-of-flight (QTOF) was 20 ppm while the product mass tolerance was $50 \mathrm{ppm}$. Filters were placed to include proteins with scores $>11$ and peptides $>6$. Additionally, the spectral peak intensity (SPI) was required to be $60 \%$ or greater. Sample run and data analysis were completed at the National Jewish Proteomics Core Facility in Denver, Colorado.

In silico protein homology and interaction modeling of CI. All bioinformatic analyses and molecular modeling simulations were performed using Accelrys Discovery Studio 3.1 (Accelrys Software; http://accelrys. com). The crystal structure coordinates of the hydrophilic domain of CI from Thermus thermophilus (PDB: 3IAM) was obtained from the protein data bank (http://www.pdb.org). The homology model of the rat CI protein sequence was constructed using the crystal structure coordinates as a template (Eswar et al., 2006). Forty-eight percent similarity was identified between the T. thermophilus and rat sequences. Modified protein structures were made by altering the side chain of wild-type (WT) residue (Arg44) to the modified form (as determined by MS). Predicted modification-induced changes in protein conformation and surface charge were determined by subjecting the WT and modified structures to the conjugate gradient minimization protocol using a CHARMM force field and the Generalized Born implicit solvent model (Feig et al., 2004). Predicted changes in the interaction between the $51 \mathrm{kDa}$ subunit and the WT or modified $75 \mathrm{kDa}$ subunit were calculated using the ZDock algorithm (Chen and Weng, 2002). The ZRank scoring function was used to calculate the interaction energies of the resulting predicted protein complexes and represents a combination of van der Waals attractive and repulsive energies, short- and long-range repulsive and attractive energies, and desolvation (Pierce and Weng, 2007). Similarity of the predicted protein complexes to the crystal structure (3IAM) was determined by calculating and the root mean square deviation (RMSD) using the crystal structure as the reference. The four predicted protein complexes with the lowest RMSD values (i.e., the highest similarity to the crystal structure) were selected from each group for comparison.

\section{Results}

To establish the temporal relationship between seizure activity and the occurrence of mitochondrial oxidative stress during the process of epileptogenesis, we monitored rats for behavioral seizures by continuous video recordings. Biochemical assessment of mitochondrial oxidative stress and enzyme activities was conducted at three time points, i.e., $48 \mathrm{~h}, 1$ week, and 6 weeks, which are associated with distinct phases of epileptogenesis, i.e., the acute, latent, and chronic periods, respectively. After injection of a single large dose of KA, severe, continuous seizure activity (SE) lasting $\sim 6 \mathrm{~h}$ was observed in rats followed by recovery. One week after KA injection, rats resumed normal behavior and showed no overt seizure activity consistent with a latent period (Dudek et al., 2002). Only rats observed to experience multiple, spontaneous convulsive seizures during the 6 week time point (chronic period), as characterized by bilateral clonus and/or loss of balance [Class IV and V seizures based on a modified Racine scale (Racine, 1972)], were considered epileptic and included in subsequent studies. Nonresponders, i.e., KA-treated rats that did not experience SE, were analyzed for evaluation of seizure-dependent effects. Saline-treated controls did not show any acute or spontaneous seizure activity. The results are consistent with our previous observations (Jarrett et al., 2008) and have allowed us to assess the consequences of mitochondrial oxidative stress during the acute, latent, and chronic stages of epileptogenesis.

\section{Carbonylation of mitochondrial proteins and specific subunits of $\mathrm{CI}$ during epileptogenesis}

One important mechanism by which ROS may alter cellular functions is via oxidative modifications of important proteins.
Protein carbonylation is typical, as a result of aging or exposure to oxidative stress. It can inhibit protein function or induce degradation and is thus considered a biomarker for oxidant-induced cellular damage (Levine and Stadtman, 2001; Dalle-Donne et al., 2003). Using a biotin-hydrazide method to detect carbonylated proteins by Western blot analysis, we determined whether carbonylation of mitochondrial proteins occurred during epileptogenesis. We observed a significant increase in total carbonylated proteins within crude mitochondria at acute and chronic time points after KA administration (Fig. $1 A$ ), which is a marker for mitochondrial oxidative stress. Carbonylation was significantly increased, as evidenced by elevated biotin immunoreactivity after derivatization of carbonyl moieties with biotin-hydrazide, by $469.4 \%$ at $48 \mathrm{~h}(p<0.001)$ and $842.2 \%$ at 6 week $(p<0.001)$ but not at 1 week $(p>0.05)$ in KA-treated rats compared with controls (Fig. $1 B$ ). In a separate study comparing rats that did not have seizures following KA treatment, i.e., nonresponders, carbonylation of mitochondrial proteins was not increased (Fig. $1 C, D)$ when compared with Ctl, demonstrating that carbonylation was not due to exposure to KA per se but a consequence of seizure activity. Furthermore, protein carbonylation was examined in mitochondria isolated from cortex and cerebellum to determine the specificity of seizure-induced oxidative stress in the KA model. Again, compared with control (saline-treated rats), marked increases in protein carbonylation were observed in the hippocampus but not cortex or cerebellum of rats treated with KA for $48 \mathrm{~h}$ ( $n=3$ /group data not shown). An additional study was conducted to assess specific changes in carbonylation to CI. Upon isolation of CI subunits, several proteins displayed elevated levels of carbonyl adducts at the $48 \mathrm{~h}$ and 6 week time points after KA ( $n=3$; data not shown). Interestingly, elevated carbonylation was observed at the $48 \mathrm{~h}$ and 6 week time points in a band corresponding to the $75 \mathrm{kDa}$ subunit (Fig. $2 \mathrm{~A}$ ). The biphasic pattern of mitochondrial protein carbonylation observed here corresponded with levels of $\mathrm{H}_{2} \mathrm{O}_{2}$ production during epileptogenesis reported in our previous study (Jarrett et al., 2008).

\section{Inhibition of mitochondrial CI activity during epileptogenesis}

Oxidative modification and inhibition of CI have been observed in neurodegenerative and psychiatric disorders, such as PD and bipolar disorder (Keeney et al., 2006; Andreazza et al., 2010). Since CI deficiency has also been observed in the seizure foci and surviving CA3 cell layer in tissue from TLE patients (Kunz et al., 2000; Lee et al., 2008), we asked whether inhibition of CI activity occurred during KA-induced epileptogenesis. An inverse correlation was observed between total mitochondrial carbonylation and CI activity (Fig. 2 B). Significant decreases of 24 and $23 \%$ in $\mathrm{CI}$ activity occurred at the acute ( $48 \mathrm{~h}$ ) and chronic (6 week) phases of epileptogenesis but not during the latent phase (1 week; Fig. 2B). To determine whether CI activity is inhibited within neuron-enriched synaptic region of the hippocampus, synaptosomes were isolated from saline- and KA-treated rats after $48 \mathrm{~h}$. Results indicated that CI activity was significantly decreased in synaptosomes after KA treatment $(\mathrm{Ctl}=203 \pm 6.804$ and $\mathrm{KA}=$ $168.0 \pm 4.429 \mathrm{U} / \mathrm{min} / \mathrm{mg}$ protein, $n=4-5, p=0.0037$, Student's $t$ test). A separate study was conducted to determine whether CI activity was altered in cortex and cerebellum in addition to the hippocampus so as to determine regional specificity of seizureinduced oxidative damage in the KA model. Comparison of hippocampus, cortex, and cerebellum revealed statistically significant decrease of CI activity in the hippocampus, but not cortex or cerebellum of rats treated with $\mathrm{KA}$ for $48 \mathrm{~h}$ (Cortex $\mathrm{Ctl}=$ 
A

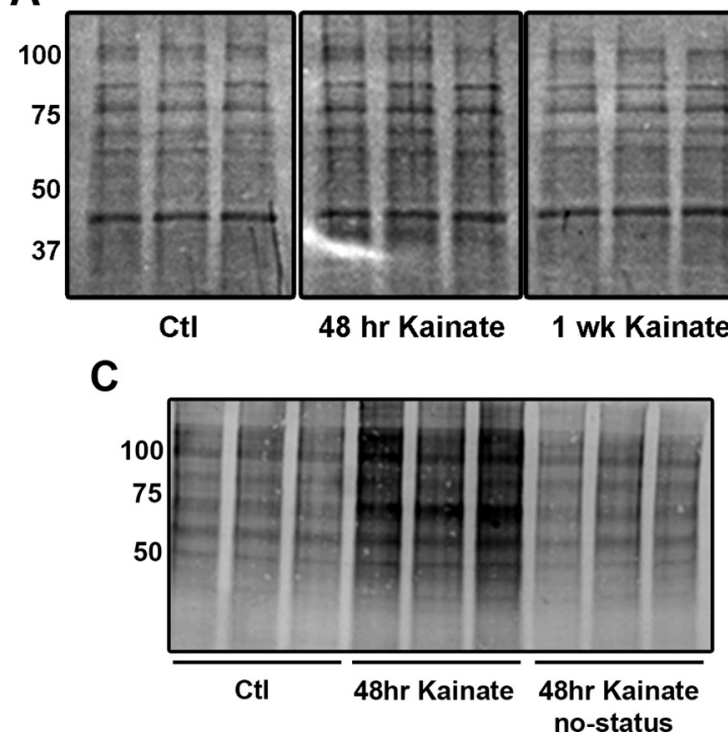

B

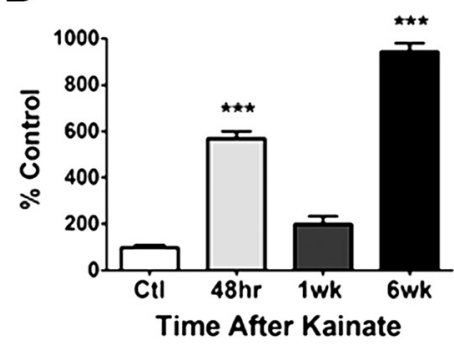

Figure 1. Elevation of carbonylated mitochondrial proteins observed in KA-treated rats at $48 \mathrm{~h}$ and 6 week time points during epileptogenesis. $\boldsymbol{A}$, Thirty micrograms of protein from saline (Ctl), $48 \mathrm{~h}, 1$ week, and 6 week KA-treated rats were assessed for carbonylation via the biotin-hydrazide method and detected by Western blot analysis; $n=3$. Band intensity correlates with carbonylated proteins in isolated crude mitochondria. B, Quantification of carbonylated proteins using NIH ImageJ 1.44 analysis software. ${ }^{* *} p<0.001$, one-way ANOVA with Dunnett's post hoc test. C, In a separate study, $10 \mu \mathrm{g}$ protein from saline (Ctl), $48 \mathrm{~h} \mathrm{KA-treated,} \mathrm{and} 48 \mathrm{~h}$ KA-treated NS rats that did not meet criteria for SE (designated as no-status or NS) were assessed for carbonylation via the biotin-hydrazide method and detected by Western blot analysis; $n=3$. D, Quantification of carbonylated proteins using NIH ImageJ 1.44 analysis software. ${ }^{* * *} p<0.001$, one-way ANOVA with Dunnett's post hoc test.

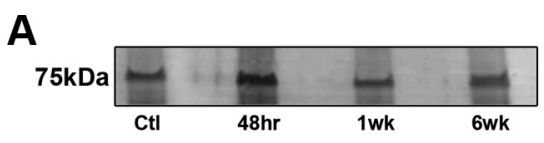

B

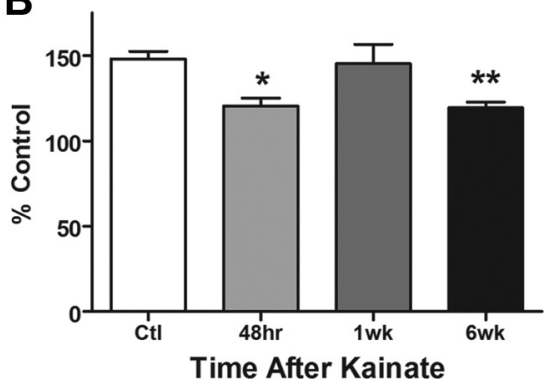

Figure 2. Increased carbonylated protein in Cl subunits and concomitant decreases in activity at $48 \mathrm{~h}$ and 6 week time points during epileptogenesis. $\boldsymbol{A}$, Representative blot (focusing on the $75 \mathrm{kDa}$ region of $\mathrm{Cl})$ of $20 \mu \mathrm{g}$ (l protein immunoprecipitated from saline $(\mathrm{Cll}), 48 \mathrm{~h}, 1$ week, and 6 week KA-treated rats was assessed for carbonylation via the biotin-hydrazide method and detected by Western blot analysis; $n=3 . B$, Cl activity measured spectrophotometrically in 100 $\mu \mathrm{g}$ mitochondria isolated from rat hippocampus, $n=6-10{ }^{*} p \leq 0.05 ;{ }^{* *} p \leq 0.01$, one-way ANOVA with Dunnett's post hoc test.

$79.03 \pm 9.474$ and $\mathrm{KA}=96.07 \pm 12.32$; Cerebellum $\mathrm{Ctl}=$ $144.9 \pm 4.542$ and $\mathrm{KA}=160.8 \pm 19.24 \mathrm{U} / \mathrm{min} / \mathrm{mg}$ protein, $n=$ $3)$. Previous work in our laboratory showed that mitochondrial complex II and III activities do not significantly change in the KA model of epileptogenesis (S. Jarrett and M. Patel, unpublished observations). Additionally, analysis of complex IV activity $48 \mathrm{~h}$, 1 week, and 6 weeks indicated no significant change in response to $\mathrm{KA}$-induced epileptogenesis $(\mathrm{Ctl}=74.38 \pm 4.636,48 \mathrm{~h} ; \mathrm{KA}=$ $65.76 \pm 4.414,1$ week $\mathrm{KA}=70.4 \pm 2.849,6$ week $\mathrm{KA}=63.64 \pm$ $7.9 \mathrm{U} / \mathrm{min} / \mathrm{mg}$ protein; $p>0.05, n=3-5 /$ group). This suggests that neuronal loss per se does not contribute to CI inhibition. Thus, specific decreases of CI activity occur during periods of carbonyl adduct formation in hippocampal mitochondria during epileptogenesis.

\section{Isolation and identification of the $75 \mathrm{kDa}$ subunit of $\mathrm{CI}$}

The $75 \mathrm{kDa}$ subunit of CI was chosen as a potential site of carbonylation, since it is the largest subunit within the hydrophilic domain of CI and has previously been shown to be carbonylated in resected brain tissue from PD patients with CI deficiency (Keeney et al., 2006). Before focusing on the $75 \mathrm{kDa}$ subunit, we first sought to improve CI subunit isolation and separation methods from crude mitochondrial preparations in saline and KAtreated rats. We were able to reliably immunoprecipitate $30 \mu \mathrm{g}$ of total CI protein and separate the subunits by SDS-PAGE. Evaluation of Coomassie-stained bands revealed no overt decreases in CI protein subunits between control and experimental groups (Fig. 3A). Initially, our goal was to identify as many CI subunits with a PMF strategy by MALDI TOF MS. Fifty percent of CI subunits were consistently identified using this technique, including the $75 \mathrm{kDa}$ subunit which corresponds to Band 1 in Figure $3 B$.

\section{Carbonylation (MCO) of Arg76 to GSA76 on the $75 \mathrm{kDa}$ subunit during epileptogenesis}

QTOF MS with electrospray ionization was used to increase total sequence coverage of the $75 \mathrm{kDa}$ subunit from $45 \%$, with MALDI TOF MS, to $62 \%$, which provided enhanced ability to measure oxidative modifications of peptides in KA-treated rats. Analysis of the MS data for the oxidation of the $75 \mathrm{kDa}$ subunit in hippocampi of saline or KA-treated rats during acute, latent, and chronic phases of epileptogenesis revealed a specific type of carbonylation classified as MCO. MCO is typically generated in the presence of oxidative stress and increased chelatable metals such as iron. MCO primarily occurs on lysine $(\mathrm{K})$ and arginine $(\mathrm{R})$ protein residues, converting them to aminoadipic semialdehyde and GSA chemical modi- 
fication products (Amici et al., 1989). Our data indicate that Arg76 is converted to GSA76 within the $75 \mathrm{kDa}$ subunit $100 \%$ of the time at acute and chronic stages of epileptogenesis. This specific modification and location was observed on the peptide, (R)LSVAGNCRMCLVEIEK(A), as shown in Table 1. Criteria for validating MS data with SpectraMill analysis software included amino acid percentage coverage $>30 \%$, SPI $>60 \%$, a quantitative match between mass to charge ratio $(\mathrm{m} / \mathrm{z})$, and the expected mass based on singly protonated ions $(\mathrm{MH}+)$, all of which are shown in Table 1 . In addition, a visual inspection of MS and MS/MS spectra was conducted (data not shown). GSA76 was observed only $25 \%$ of the time during the latent period, which is consistent with slight increases in mitochondrial carbonylation at 1 week (Fig. 1). Control animals did not have the GSA76 modification although the peptide was present. It should be noted that protein sequence coverage was similar between saline and experimental groups (data not shown). These data parallel mitochondrial carbonylation and CI inhibition data, indicating that MCO of Arg76 to GSA76 may have a functional impact on CI during epileptogenesis.

\section{Functional consequences of carbonylation on the $75 \mathrm{kDa}$ subunit during epileptogenesis}

To determine the role of Arg76 in normal CI function we first examined the evolutionary conservation of this residue via a multiple sequence alignment between the $75 \mathrm{kDa}$ subunit amino acid sequences of bacteria ( $T$. thermophilus), mouse (Mus musculus), rat (Rattus norvegicus), cow (Bos taurus), and human (Homo sapiens) (Fig. 4A). Arg76, which corresponds to Arg44 in the bacterial sequence, is $100 \%$ conserved across all species examined, suggesting the importance of this residue to the proper function of CI. To assess functional consequences of formation of a GSA at this position during epileptogenesis we constructed homology models of the individual subunits comprising the rat CI hydrophilic domain based on the bacterial crystal structure template from T. thermophilus (Fig. 4B). The individual homology models of the rat CI hydrophilic subunits were assembled into their tertiary form by molecular overlay with the bacterial crystal structure (Fig. 4C). Sequence alignments of the hydrophilic subunits of CI between bacterial and rat species revealed an overall $48 \%$ sequence similarity among the entire hydrophilic domain of CI, while the $75 \mathrm{kDa}$ subunit alone exhibited a $43 \%$ similarity between the two species. The alignment between the bacterial and rat CI indicated that Arg44 is the bacterial ortholog of Arg76 in the rat $75 \mathrm{kDa}$ sequence. Both Arg44 in the crystal structure and Arg76 in our homology model are solvent-accessible residues located at the interaction interface between the 75 and $51 \mathrm{kDa}$ subunits, in close proximity to the NADH binding site and the first Fe-S center involved in electron transfer (Fig. $4 B, C$ ). To examine the predicted alterations in the protein conformation of the complex in response to the GSA modification, the WTand GSA-modified crystal structures of CI from T. thermophilus (3IAM) were subjected to energy minimization using the generalized Born implicit solvent model. The modification induced little change in the conformation of the $75 \mathrm{kDa}$ sub- unit within the complex but resulted in substantial predicted shifts in the positions of residues Ile394-Ala405 and His350Arg360 of the $51 \mathrm{kDa}$ subunit (Fig. 4D). Additionally, the proximal $\mathrm{Fe}-\mathrm{S}$ center was observed to shift position away from the NADH binding site (Fig. $4 D$ ). The modification-induced changes in protein conformation of the $51 \mathrm{kDa}$ subunit were sufficient to significantly impair the predicted interaction between the 75 and $51 \mathrm{kDa}$ subunits by $15 \%(p=0.047)$ (Fig. $4 E$ ). Compared with the WT structure (Fig. $5 A$ ), the interface of the GSA-modified $75 \mathrm{kDa}$ subunit involved in the interaction with the $51 \mathrm{kDa}$ subunit also exhibited substantial predicted changes in molecular surface topology, as well as interpolated surface charge at the Arg site from positive (blue) to negative (red) (Fig. 5B).Together, these data demonstrate that specific MCO-induced carbonylation of Arg76 has the capacity to alter CI protein structure and function during acute and chronic stages of epileptogenesis.

\section{Discussion}

This study demonstrates for the first time an oxidative PTM of $\mathrm{CI}$, an important component of the mitochondrial ETC complex, in epileptogenesis. The data support the conclusion that oxidative modification via carbonylation of the $75 \mathrm{kDa}$ subunit of CI occurs in a biphasic manner during epileptogenesis, coincident with carbonylation of mitochondrial proteins and inhibition of CI activity. The nature of the post-translational oxidative modification was identified as a specific metal catalyzed carbonylation of the $75 \mathrm{kDa}$ subunit, converting Arg76 to GSA76. Our molecular modeling studies indicate that carbonylation of the $75 \mathrm{kDa}$ is predicted to alter its surface structure and charge at the interaction interface within the $\mathrm{CI}$ active site, its interaction with the 51 $\mathrm{kDa}$ subunit of $\mathrm{CI}$, and the position of nearby Fe-S centers proximal to the CI active site, which can impair electron transfer from $\mathrm{NADH}$ to the proximal Fe-S center, and thus CI function. Collectively these data identify a post-translational oxidative lesion on Arg76 in an important subunit of mitochondrial CI during epileptogenesis.

Previous work has demonstrated increased mitochondrial ROS production during the acute and chronic phases of epileptogenesis (Jarrett et al., 2008; Waldbaum et al., 2010). Here we demonstrate a specific ROS-mediated oxidative PTM in the 75 $\mathrm{kDa}$ subunit of CI during the acute and chronic phases of epilepsy development, which can impair mitochondrial function during epileptogenesis. The significance of carbonylation and inhibition of CI is twofold. First, CI is not only a target of oxidative stress via 
Table 1. Mass spectrometry identification of carbonylation on the $75 \mathrm{kDa}$ subunit

\begin{tabular}{llllllll}
\hline & AA\# & AA \% coverage & SPI \% & Peptide sequence & Modification & m/z (Da) measured & MH+ (Da) matched \\
\hline Control & 69 & 48 & 83.1 & (R)LSVAGNCRmCLVEIEK(A) [100\%] & m-0xidized methionine & 594.2989 & 1780.8803 \\
48 h KA & 69 & 55 & 77.2 & (R)LSVAGNcrMCLVEIEK(A)[100\%] & c-Carbamidomethyl; r-GSA & 593.6242 & 1778.8673 \\
1 week KA & 69 & 48 & 83.1 & (R)LSVAGNCRmCLVEIEK(A) [75\%] & m-Oxidized methionine & 594.2989 & 1780.8803 \\
1 week KA & 69 & 38 & 64.3 & (R)LSVAGNcrMCLVEIEK(A) [25\%] & c-Carbamidomethyl; r-GSA & 593.623 & 1778.8682 \\
6 week KA & 69 & 62 & 84 & (R)LSVAGNcrMCLVEIEK(A) [100\%] & c-Carbamidomethyl; r-GSA & 593.6244 & 1778.8674 \\
\hline
\end{tabular}

A GSA (carbonylation) PTM was identified by QTOF MS analysis on a single peptide, (R)LSVAGNCRMCLVEIEK(A), at arginine (r) 76 . This modification occurred $100 \%$ of the time at $48 \mathrm{~h}$, $25 \%$ at 1 week, and $100 \%$ at 6 week during epileptogenesis; $n=4$ rats/group. $A A=$ amino acid; Da, Dalton; $\mathrm{KA}$, kainate; $\mathrm{MH}+=$ expected protonated ions; $\mathrm{m} / \mathrm{z}=$ mass to charge ratio; $\mathrm{SPI}=$ spectral peak intensity.
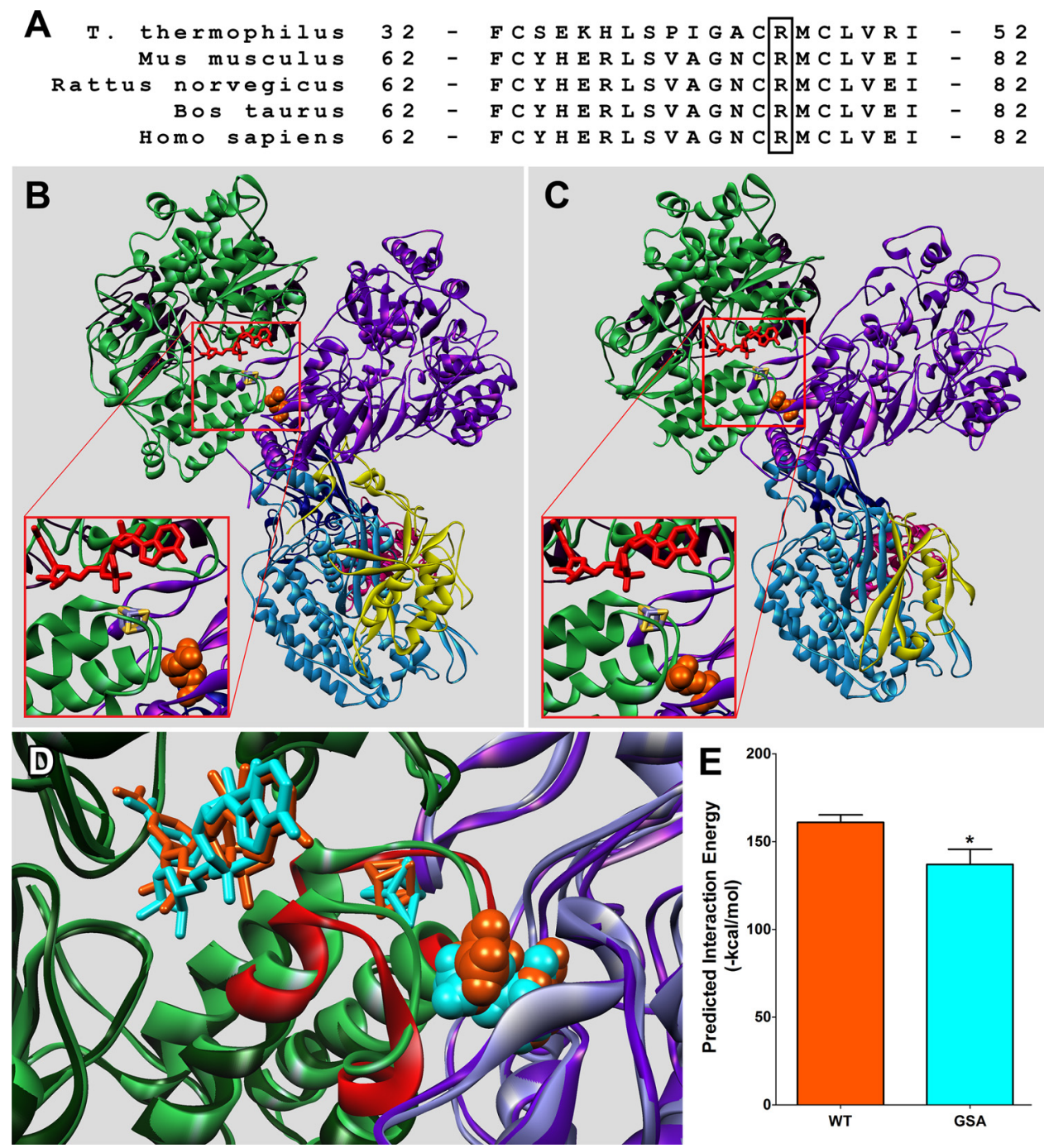

Figure 4. Location of the GSA-modified residue and the predicted modification-induced alterations in protein conformation and interaction energy. $A$, Multiple sequence alignment between bacteria (T. thermophilus), mouse (M. musculus), rat (R. norvegicus), cow (B. taurus), and human (H. sapiens) to show $100 \%$ conservation of arginine across multiple species. $\boldsymbol{B}$, Crystal structure of the hydrophilic domain of Cl from T. thermophilus (PDB ID: 3IAM). C, Protein homology model of the rat ortholog constructed using 3IAM as a template. The modified residue is depicted as orange spheres with bound $\mathrm{NADH}$ in red and the individual Cl subunits colored as follows: $75 \mathrm{kDa}$, purple; $51 \mathrm{kDa}$, green; $24 \mathrm{kDa}$, maroon; $49 \mathrm{kDa}$, light blue; $30 \mathrm{kDa}$, dark blue; TYKY, pink; and PSST, yellow. Insets, Close-ups of the NADH binding site, the initial Fe-S center involved in electron transfer (purple and yellow sticks), and Arg44/Arg76 (orange spheres). D, Overlay of minimized WT and GSA-modified 3IAM structures. WT 51 and $75 \mathrm{kDa}$ subunits are light green and light purple; the structures of the modified 51 and $75 \mathrm{kDa}$ subunits are dark green and dark purple. NADH, the first Fe-S center, and the GSA-modified residue are colored orange for the WT structure and cyan for the modified. Predicted conformational changes observed in the $51 \mathrm{kDa}$ subunit in response to the modification are colored red. $E$, Predicted energy of interaction between the 51 and $75 \mathrm{kDa}$ subunits of the WT and the modified (GSA) structures. ${ }^{*} p<0.05$ with Student's $t$ test.

PTMs, but it is also a source of superoxide production when inhibited, generating excess reactive species and further mitochondrial and cellular damage (Kudin et al., 2004; Fato et al., 2008; Hirst, 2010; Murphy, 2009). Therefore, CI may be both a source and target of ROS creating a vicious cycle in which seizures result in ROS production leading to oxidative inactivation of CI, which in turn results in increased superoxide formation. Second, specific CI deficiency has been observed in tissue resected from TLE patients (Kunz et al., 2000), but the mechanism by which CI inhibition occurred was unknown. In the latter study, reduction in CI activity was postulated to occur due to decreases in mitochondrial DNA copy number and a concomitant downregula- 

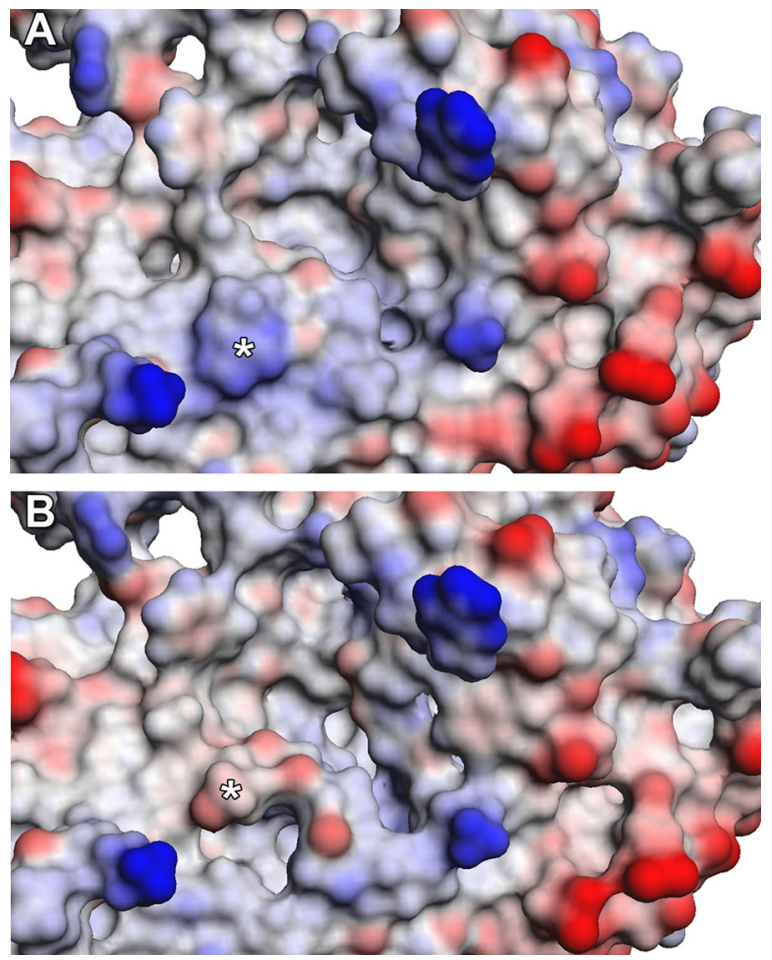

Figure 5. Predicted structural and surface charge alterations to the interaction interface of the $75 \mathrm{kDa}$ subunit in response to carbonylation of Arg44. $A, B$, Molecular surface representation of the interaction interface of the $75 \mathrm{kDa}$ subunit from the energy minimized crystal structure of $T$. thermophilus of the WT $(\boldsymbol{A})$ and GSA-modified form $(\boldsymbol{B})$. Interpolated surface charge is indicated as a range from positive (blue) to neutral (white) to negative (red). The asterisks denote the positions of Arg44 and the GSA-modified form.

tion of oxidative phosphorylation enzymes encoded by mitochondrial DNA. Our data in the KA model of TLE provides a potential mechanism for the loss of CI activity through PTM of the enzyme by ROS. The biphasic occurrence of CI modification during the acute and chronic phases of KA-induced epileptogenesis, but not the latent period, is consistent with the time course of increased mitochondrial $\mathrm{H}_{2} \mathrm{O}_{2}$ production observed previously (Jarrett et al., 2008; Waldbaum et al., 2010) and with carbonylation of mitochondrial proteins (Fig. 1) in the hippocampus. The absence of mitochondrial carbonylation in rats unresponsive to $\mathrm{KA}$ indicates that mitochondrial oxidative damage is a consequence of seizure activity rather than KA exposure per se. To date, CI modification by glutathionylation, nitrosylation, and carbonylation has been suggested to inhibit function of the enzyme (Taylor et al., 2003; Dahm et al., 2006; Andreazza et al., 2010). In this study, we report the evidence that carbonylation is the cause of CI inhibition during epileptogenesis. In contrast with glutathionylation and nitrosylation, which are reversible modifications, carbonylation is a covalent oxidative modification and is therefore capable of permanently damaging the protein. As previously noted, CI carbonylation has been observed in tissue resected from PD patients (Keeney et al., 2006) and the pattern of subunit oxidation was similar to studies using rotenone to pharmacologically block CI function. The latter study suggested that only ROS generated from internal CI inhibition could create this pattern of CI carbonylation and proposed the significance of CI modifications during disease states. However, the specific location and type of carbonylation was not identified. Proteinspecific carbonyl adducts are difficult to detect in vivo due to their low abundance, turnover, and loss during sample preparation and analysis (Berlett and Stadtman, 1997; Fritz and Petersen, 2011; Møller et al., 2011). Furthermore, endogenous carbonylation to mitochondrial ETC complexes has been reported and highlights the importance of distinguishing between disease-state modifications versus those resulting from normal ROS production (Choksi et al., 2004).

This study suggests that the mechanism for CI dysfunction is potentially due to specific MCO of Arg76 to a GSA76 in the $75 \mathrm{kDa}$ subunit. MCO is a major route for protein carbonyl formation in biological samples and can yield highly reactive carbonyl derivatives (i.e., GSA products), which can negatively impact protein function, turnover, or DNA binding (Requena et al., 2001). Although MCO may not be the most common type of carbonyl addition in vivo, the probability of its identification in this study is elevated due to our previous reports that mitochondrial Fenton reactants, $\mathrm{H}_{2} \mathrm{O}_{2}$ and $\mathrm{Fe}^{2+}$, are increased in the KA model (Jarrett et al., 2008; Liang et al., 2008). These data suggest that MCO products may exist in other neurological conditions with increased mitochondrial oxidative stress and elevated redox active chelatable metals such as PD (Gerlach et al., 1994; Liang and Patel, 2004; Lee and Andersen, 2010).

The location of GSA76 is also a critical component for understanding the mechanism of CI inhibition by MCO. The structure of CI implies that the catalytic center is relatively shielded from ROS and the majority of PTMs occur on exposed surfaces of subunits within the hydrophilic arm of the complex (Murphy, 2009). Molecular modeling of the hydrophilic arm of CI in rat shows that Arg76 (or GSA76) is uniquely situated near the $\mathrm{N}$ terminus of the $75 \mathrm{kDa}$ subunit; this location is not only close to the active site, it is also surface exposed, increasing the probability for its modification. Sequence alignments of the $75 \mathrm{kDa}$ subunit between bacteria, mouse, rat, cow, and human species show that this arginine and its flanking amino acids are 100\% conserved, indicating both the importance of this region to CI function and its potential to be modified in multiple species during oxidative stress. A reactive GSA product near the active site of CI (1) changed the spatial orientation of the adjacent $51 \mathrm{kDa}$ subunit containing the NADH binding site and (2) changed the position of the proximal Fe-S, which may impact electron transfer. The modified Arg76 residue is located in close proximity to the $\mathrm{NADH}$ binding site; however, the modification had no effect on the predicted binding of NADH (data not shown), suggesting that the observed decrease in in vivo activity is not due to altered affinity for NADH. Given the substantial predicted changes to the protein surface topology and charge in response to formation of the GSA modification, it is not surprising that the modification is also predicted to significantly impair the interaction between the 51 and $75 \mathrm{kDa}$ subunits. While this does not necessarily suggest that the two subunits are dissociated as a result of modification, results may indicate a decrease in the cohesiveness of the active site that may lower CI activity without altering the affinity for $\mathrm{NADH}$ per se. Interestingly, the modification induced a predicted shift in the position of the Fe-S center involved in the initial electron transfer from $\mathrm{NADH}$ that resulted in an increase in the distance between the two molecules. This suggests the possibility that the changes in activity in response to the modification may be due to impairment of the initial transfer of electrons from $\mathrm{NADH}$ to the Fe-S center.

While we chose to focus our efforts on the $75 \mathrm{kDa}$ subunit due to its location within the mitochondrial matrix (i.e., close proximity to reactive species), its well documented capacity for PTM, 
and its critical role in the CI active site (Hirst et al., 2003), it is likely that additional CI subunits are carbonylated. In addition, our increased detection of total carbonylated mitochondrial proteins does not indicate that all modifications are necessarily the result of MCO. Biotin-hydrazide, which was used to detect carbonylated proteins, can label various aldehyde and ketone carbonyl groups that may be the products of diverse oxidative reactions. We recognize that the discovery of adducts can be difficult in vivo and determination of all PTMs of the CI subunits during epileptogenesis may require more sophisticated detection techniques.

In summary, we have shown that MCO within the $\mathrm{CI}$ active site is occurring during epileptogenesis. Persistent CI inhibition during the chronic phase of epileptogenesis can ultimately inhibit mitochondrial ATP production leading to a bioenergetic crisis. Multiple studies have shown sensitivity of ETC complexes to oxidative stress (Zhang et al., 1990; Cadenas and Davies, 2000). ATP depletion can affect various cellular functions, including neuronal excitability, by interfering with ATP-dependent ion channels that maintain neuronal membrane potential leading to chronic mitochondrial dysfunction (Jamme et al., 1995; Nicholls and Ward, 2000; Fighera et al., 2006). These observations suggest that oxidative damage and CI carbonylation may have a mechanistic role in epileptogenesis be a future therapeutic target for the prevention of acquired epilepsies.

\section{References}

Amici A, Levine RL, Tsai L, Stadtman ER (1989) Conversion of amino acid residues in proteins and amino acid homopolymers to carbonyl derivatives by metal-catalyzed oxidation reactions. J Biol Chem 264:3341-3346.

Andersen JK (2004) Oxidative stress in neurodegeneration: cause or consequence? Nat Med 10 [Suppl] S18-S25.

Andreazza AC, Shao L, Wang JF, Young LT (2010) Mitochondrial complex I activity and oxidative damage to mitochondrial proteins in the prefrontal cortex of patients with bipolar disorder. Arch Gen Psychiatry 67:360-368.

Beal MF (2005) Mitochondria take center stage in aging and neurodegeneration. Ann Neurol 58:495-505.

Berlett BS, Stadtman ER (1997) Protein oxidation in aging, disease, and oxidative stress. J Biol Chem 272:20313-20316.

Birch-Machin MA, Turnbull DM (2001) Assaying mitochondrial respiratory complex activity in mitochondria isolated from human cells and tissues. Methods Cell Biol 65:97-117.

Cadenas E, Davies KJ (2000) Mitochondrial free radical generation, oxidative stress, and aging. Free Radic Biol Med 29:222-230.

Chen R, Weng Z (2002) Docking unbound proteins using shape complementarity, desolvation, and electrostatics. Proteins 47:281-294.

Choksi KB, Boylston WH, Rabek JP, Widger WR, Papaconstantinou J (2004) Oxidatively damaged proteins of heart mitochondrial electron transport complexes. Biochim Biophys Acta 1688:95-101.

Codreanu SG, Zhang B, Sobecki SM, Billheimer DD, Liebler DC (2009) Global analysis of protein damage by the lipid electrophile 4-hydroxy-2nonenal. Mol Cell Proteomics 8:670-680.

Dahm CC, Moore K, Murphy MP (2006) Persistent S-nitrosation of complex I and other mitochondrial membrane proteins by S-nitrosothiols but not nitric oxide or peroxynitrite: implications for the interaction of nitric oxide with mitochondria. J Biol Chem 281:10056-10065.

Dalle-Donne I, Giustarini D, Colombo R, Rossi R, Milzani A (2003) Protein carbonylation in human diseases. Trends Mol Med 9:169-176.

Dalle-Donne I, Aldini G, Carini M, Colombo R, Rossi R, Milzani A (2006) Protein carbonylation, cellular dysfunction, and disease progression. J Cell Mol Med 10:389-406.

Danielson SR, Andersen JK (2008) Oxidative and nitrative protein modifications in Parkinson's disease. Free Radic Biol Med 44:1787-1794.

Dudek FE, Hellier JL, Williams PA, Ferraro DJ, Staley KJ (2002) The course of cellular alterations associated with the development of spontaneous seizures after status epilepticus. Prog Brain Res 135:53-65.

Eswar N, Webb B, Marti-Renom MA, Madhusudhan MS, Eramian D, Shen
MY, Pieper U, Sali A (2006) Comparative protein structure modeling using Modeller. Curr Protoc Bioinformatics Chapter 5:Unit 5.6.

Fato R, Bergamini C, Leoni S, Lenaz G (2008) Mitochondrial production of reactive oxygen species: role of complex I and quinone analogues. Biofactors 32:31-39.

Feig M, Onufriev A, Lee MS, Im W, Case DA, Brooks CL 3rd (2004) Performance comparison of generalized born and Poisson methods in the calculation of electrostatic solvation energies for protein structures. J Comput Chem 25:265-284.

Fighera MR, Royes LF, Furian AF, Oliveira MS, Fiorenza NG, Frussa-Filho R, Petry JC, Coelho RC, Mello CF (2006) GM1 ganglioside prevents seizures, $\mathrm{Na}+, \mathrm{K}+$-ATPase activity inhibition and oxidative stress induced by glutaric acid and pentylenetetrazole. Neurobiol Dis 22:611-623.

Fritz KS, Petersen DR (2011) Exploring the biology of lipid peroxidationderived protein carbonylation. Chem Res Toxicol 24:1411-1419.

Galligan JJ, Smathers RL, Fritz KS, Epperson LE, Hunter LE, Petersen DR (2012) Protein carbonylation in a murine model for early alcoholic liver disease. Chem Res Toxicol 25:1012-1021.

Gerlach M, Ben-Shachar D, Riederer P, Youdim MB (1994) Altered brain metabolism of iron as a cause of neurodegenerative diseases? J Neurochem 63:793-807.

Hirst J (2010) Towards the molecular mechanism of respiratory complex I. Biochem J 425:327-339.

Hirst J, Carroll J, Fearnley IM, Shannon RJ, Walker JE (2003) The nuclear encoded subunits of complex I from bovine heart mitochondria. Biochim Biophys Acta 1604:135-150.

Jamme I, Petit E, Divoux D, Gerbi A, Maixent JM, Nouvelot A (1995) Modulation of mouse cerebral $\mathrm{Na}+, \mathrm{K}(+)$-ATPase activity by oxygen free radicals. Neuroreport 7:333-337.

Jarrett SG, Liang LP, Hellier JL, Staley KJ, Patel M (2008) Mitochondrial DNA damage and impaired base excision repair during epileptogenesis. Neurobiol Dis 30:130-138.

Keeney PM, Xie J, Capaldi RA, Bennett JP Jr (2006) Parkinson's disease brain mitochondrial complex I has oxidatively damaged subunits and is functionally impaired and misassembled. J Neurosci 26:5256-5264.

Kudin AP, Bimpong-Buta NY, Vielhaber S, Elger CE, Kunz WS (2004) Characterization of superoxide-producing sites in isolated brain mitochondria. J Biol Chem 279:4127-4135.

Kunz WS, Kudin AP, Vielhaber S, Blümcke I, Zuschratter W, Schramm J, Beck H, Elger CE (2000) Mitochondrial complex I deficiency in the epileptic focus of patients with temporal lobe epilepsy. Ann Neurol 48:766-773.

Lee DW, Andersen JK (2010) Iron elevations in the aging Parkinsonian brain: a consequence of impaired iron homeostasis? J Neurochem 112:332-339.

Lee YM, Kang HC, Lee JS, Kim SH, Kim EY, Lee SK, Slama A, Kim HD (2008) Mitochondrial respiratory chain defects: underlying etiology in various epileptic conditions. Epilepsia 49:685-690.

Levine RL, Stadtman ER (2001) Oxidative modification of proteins during aging. Exp Gerontol 36:1495-1502.

Liang LP, Patel M (2004) Iron-sulfur enzyme mediated mitochondrial superoxide toxicity in experimental Parkinson's disease. J Neurochem 90:1076-1084.

Liang LP, Patel M (2006) Seizure-induced changes in mitochondrial redox status. Free Radic Biol Med 40:316-322.

Liang LP, Jarrett SG, Patel M (2008) Chelation of mitochondrial iron prevents seizure-induced mitochondrial dysfunction and neuronal injury. J Neurosci 28:11550-11556.

Liang LP, Waldbaum S, Rowley S, Huang TT, Day BJ, Patel M (2012) Mitochondrial oxidative stress and epilepsy in SOD2 deficient mice: attenuation by a lipophilic metalloporphyrin. Neurobiol Dis 45:1068-1076.

Møller IM, Rogowska-Wrzesinska A, Rao RS (2011) Protein carbonylation and metal-catalyzed protein oxidation in a cellular perspective. J Proteomics 74:2228-2242.

Murphy MP (2009) How mitochondria produce reactive oxygen species. Biochem J 417:1-13.

Nicholls DG, Ward MW (2000) Mitochondrial membrane potential and neuronal glutamate excitotoxicity: mortality and millivolts. Trends Neurosci 23:166-174.

Pierce B, Weng Z (2007) ZRANK: reranking protein docking predictions with an optimized energy function. Proteins 67:1078-1086. 
Racine RJ (1972) Modification of seizure activity by electrical stimulation. I. After-discharge threshold. Electroencephalogr Clin Neurophysiol 32:269-279.

Requena JR, Chao CC, Levine RL, Stadtman ER (2001) Glutamic and aminoadipic semialdehydes are the main carbonyl products of metalcatalyzed oxidation of proteins. Proc Natl Acad Sci U S A 98:69-74.

Schapira AH (1999) Mitochondrial involvement in Parkinson's disease, Huntington's disease, hereditary spastic paraplegia and Friedreich's ataxia. Biochim Biophys Acta 1410:159-170.

Schilling B, Bharath MMS, Row RH, Murray J, Cusack MP, Capaldi RA, Freed CR, Prasad KN, Andersen JK, Gibson BW (2005) Rapid purification and mass spectrometric characterization of mitochondrial NADH dehydrogenase (Complex I) from rodent brain and a dopaminergic neuronal cell line. Mol Cell Proteomics 4:84-96.

Shevchenko A, Tomas H, Havlis J, Olsen JV, Mann M (2006) In-gel diges- tion for mass spectrometric characterization of proteins and proteomes. Nat Protoc 1:2856-2860.

Taylor ER, Hurrell F, Shannon RJ, Lin TK, Hirst J, Murphy MP (2003) Reversible glutathionylation of complex I increases mitochondrial superoxide formation. J Biol Chem 278:19603-19610.

Tieu K, Perier C, Caspersen C, Teismann P, Wu DC, Yan SD, Naini A, Vila M, Jackson-Lewis V, Ramasamy R, Przedborski S (2003) D-betahydroxybutyrate rescues mitochondrial respiration and mitigates features of Parkinson disease. J Clin Invest 112:892-901.

Waldbaum S, Liang LP, Patel M (2010) Persistent impairment of mitochondrial and tissue redox status during lithium-pilocarpine-induced epileptogenesis. J Neurochem 115:1172-1182.

Zhang Y, Marcillat O, Giulivi C, Ernster L, Davies KJ (1990) The oxidative inactivation of mitochondrial electron transport chain components and ATPase. J Biol Chem 265:16330-16336. 